\title{
Observations of $\mathbf{U}$ Leporis
}

\section{Larson S. Pavey, Tyler Z. Smith, Samuel G. Wyss, and Matthew P. PERKINS COPPOLA}

Corresponding author: perkinsm@pfw.edu

U Leporis (U Lep) is a pulsating variable that demonstrates periodic variation in apparent magnitude consistent with RR Lyrae variable stars. Images were acquired by the $\mathbf{0 . 4}$ meter telescopes of the Las Cumbres Observatory Global Telescope network in the visible (B, V) and near infrared ( $\mathrm{i}$ and $\mathrm{z}$ ) over a two week period in October 2019 and analyzed using Astrosource. Light curves were constructed for each of the four bandpasses, yielding a mean period of $0.581 \pm 0.011$ days. The middle apparent magnitudes determined for the $\mathrm{B}, \mathrm{V}, \mathrm{i}$, and $\mathrm{z}$ filters were $10.75,10.55,10.30$, and 10.35 respectively. Color excess E(B-V) was determined by two methods (1) minimizing variance in the calculated distances for the $V$, $i$, and $z$ bands (0.065) and (2) galactic dust (0.029), with the second method preferred. Distances in parsecs to U Lep were determined for the $\mathrm{V}(968 \pm 38), \mathrm{i}(932 \pm 33)$, and $\mathrm{z}(951 \pm 38)$ filters. The average distance of $950 \pm 21$ compares favorably to that obtained by Klein $(977 \pm 8 \mathrm{pc})$ and less so to Gaia DR2 (1076 $\pm 36 \mathrm{pc})$.

(ㅁ)(요 2021 Astronomy Theory, Observations and Methods Journal

Keywords: Variables, RR Lyrae, notices - miscellaneous - catalogs - surveys

https://doi.org/10.32374/atom.2020.2.3

\section{INTRODUCTION}

U Leporis (U Lep) is a variable star which lies within the Milky Way Galaxy. U Leporis has a right ascension of $04 \mathrm{~h} 56 \mathrm{~m} 17.96$ and Declination of $-21^{\circ} 13^{\prime} 01.5$. This star is in the constellation of Lepus located just south of the celestial equator. Another name for $\mathrm{U}$ Lep is HIP22952 from the Hipparcos Star Catalogue.

RR Lyrae stars (RRL) are understood to be lowmass ( 0.6 to $0.8 \mathrm{M}_{\odot}$ ) stars found at the intersection of the horizontal branch (HB) and the instability strip of the Hertzspring-Russell diagram (Catelan \& Smith, 2015). These older Population II (> 10 Gyr) giant (4-6 $\mathrm{R}_{\odot}$ ) stars, post-helium flash, are able to fuse helium in their cores as hydrogen fusion occurs in a shell surrounding the core. RR Lyrae stars were first used to determine the distance to globular clusters, though today more RR Lyrae stars are known in other parts of the Milky Way galaxy and are used to study the structure of the Galactic halo (Keller, Murphy, Prior, DaCosta, \& Schmidt, 2008; Hernitschek et al., 2018; Iorio \& Belokurov, 2018; Wegg, Gerhard, \& Bieth, 2019), bulge (Du, Mao, Athanassoula, Shen, \& Pietrukowicz, 2020; Soszyński et al., 2019), and the thick disk (Mateu \& Vivas, 2018). Others are using RRL to study metallicity and other details within nearby galaxies (Clementini et al., 2001; Sarajedini, Barker, Geisler, Harding, \& Schommer, 2006). 
RRL pulsate radially with periods between 1.5 to 24 hours and are divided into two classes. RRab stars pulsate in the fundamental radial mode with a light curve resembling an inverted sawtooth wave, rising rapidly then gradually fading to minimum. RRc stars pulsate in the first-overtone radial mode, producing a light curve resembling a sinusoidal pattern. The pulsation in both types is the result of layers of stellar material falling inward, compressing layers of gases found deeper within the star. At some depth a layer of hydrogen gas partially ionizes rather than increasing in temperature. Deeper still inside the star, compression forms a layer of partially ionized helium II. The opacity $\kappa$ of these layers is directly proportional to the density $\rho$ and inversely proportional to the temperature $\mathrm{T}$ raised to the 3.5 power, a relationship known as Kramer's Law.

$$
\kappa \propto \frac{\rho}{T^{3.5}}
$$

The steady temperature and increasing density of these partially ionized layers increases the opacity, allowing them to act as a blanket over the layers of gas beneath. Contraction continues until a build up of heat under the blanketed areas forces an expansion. During this outward movement the blanket expands, cools, and becomes less opaque, allowing the trapped heat to escape outward. This mechanism causes the radius of the star to vary, thus varying the surface area and luminosity of the star.

When the effective surface temperature of the star is between $5500 \mathrm{~K}$ and $7500 \mathrm{~K}$, referred to as the "instability strip," the location of these partially ionized layers of hydrogen and helium II is just right to drive stable periodic oscillations. Hotter stars form partially ionized layers too close to the surface, while cooler stars form partial ionization zones deeper in the star, where convection prevents heat from building up to cause expansion.

RRL stars are useful standard candles for determining distances but, unlike Cepheid variables, the period-luminosity relation they display is not so straightforward (Catelan, Pritzl, \& Smith, 2004). It appears the metallicity $[\mathrm{Fe} / \mathrm{H}]$ of the star must be factored into the determination of distance. Until the recent Gaia mission, distances to most known RRL were too far to be determined accurately by spectroscopic parallax (Catelan \& Smith, 2015). Previous calibrations derived from the Sloan Digital Sky Survey (Cáceres \& Catelan, 2008) for the near infrared i and $\mathrm{z}$ bandpasses suggest a reliable period-luminosity- metallicity relationship exists. This research project observes a single RRL at B, V, i, z bandpasses to compare the calibrations to parallax measures determined by Gaia and previous observations.

$\mathrm{U}$ Lep is a class RRab variable. Previous light curve measurements using Hipparcos data determined a period for U Lep of 0.581236 days (Wu, Qiu, Deng, $\mathrm{Hu}, \&$ Zhao, 2006) to 0.581479 days (Feast, Laney, Kinman, Van Leeuwen, \& Whitelock, 2008). Klein et al. (2011) used this period and the preliminary data release of the Wide-field Infrared Survey Explorer (WISE) to determine a distance of 976.8 pc to U Lep. Gaia DR2 (A. Brown et al., 2018) as accessed through Simbad (Wenger et al., 2000) yielded a corrected parallax of $0.9298 \pm 0.0276$ mas, for a distance of $1076 \pm 36 \mathrm{pc}$.

\section{OBSERVATIONS}

The Las Cumbres Observatory Global Telescope Network (LCOGT) was used to observe the target variable star, $\mathrm{U}$ Lep, in the $\mathrm{B}, \mathrm{V}, \mathrm{i}$, and $\mathrm{z}$ bandpasses. The 0.4-meter SBIG telescopes at multiple LCOGT nodes provided images with a field of view of 19'x29' (T. Brown et al., 2013) (https://lco.global/observatory/telescopes/0$4 \mathrm{~m} /$ ). Figure 1 is a representative sample from the data set.

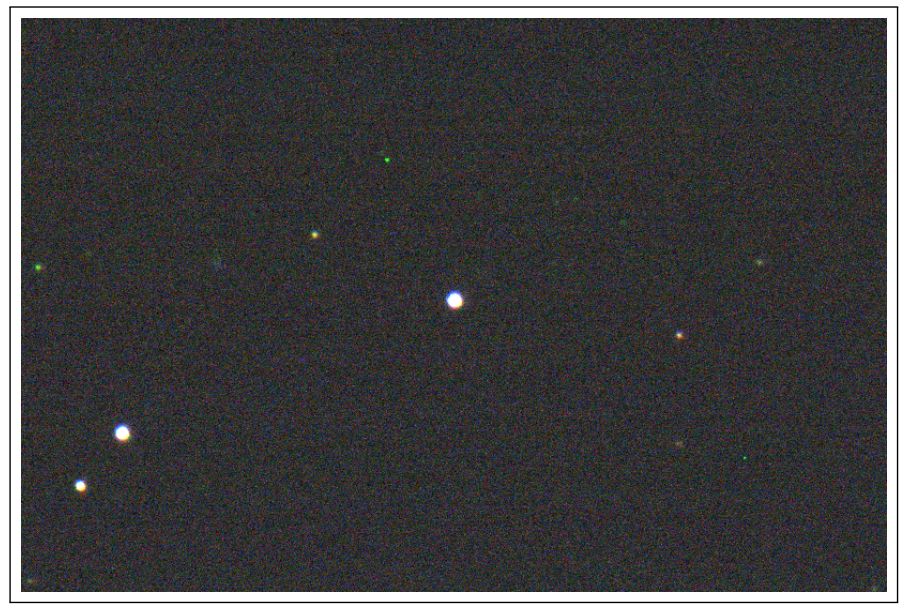

Fig. 1. A picture of U Leporis (center) taken with the 0.4-meter SBIG

First, a set of test images was obtained to determine the optimal exposure time to view U Lep for each of the four bandpasses. Each test image was examined in AstroImageJ, using the aperture tool to measure the number of counts for the target star. The number of counts for each pixel is proportional to 
the number of photons received during the exposure. Incoming photons strike the CCD, liberating electrons (e-) via the photoelectric effect, which are then collected by a capacitor. The voltage of the capacitor is converted by an Analog-To-Digital unit. The SBIG STL-6303 at the LCOGT were set for a gain (e-/ADU) of 1.6. Remembering that U Lep varies in magnitude, an acceptable range of counts was established to be between 25,000 and 250,000 and the value of 200,000 was chosen for determining the optimal exposure time. For each bandpass, the ratio of the optimal and test image exposure times was set equal to the ratio of the desired counts $(200,000)$ and those measured for the test image. The exposure times used for each band are summarized in Table 1.

Table 1. Exposure times for different color bands.

\begin{tabular}{ccc}
\hline \hline Band & Wavelength Center $(\AA)$ & Exposure (s) \\
\hline B & 4361 & 28.4 \\
V & 5448 & 12 \\
i & 7545 & 16.2 \\
Z & 8700 & 62 \\
\hline
\end{tabular}

Data was collected over the course of twelve days from October 02 to October 14, 2019. The LCOGT was used to request 75 observations of our target star. The goal was to have at least 50 points of data to evaluate for each bandpass. Requesting more observations than the goal was necessary for two reasons. First, there were windows of time during which an observation might not be able to be scheduled. Despite the availability of multiple telescopes across the globe, the target star might be too low on the horizon to be viewed by any telescope in the network. Of the 75 requested observations, only 59 were able to be scheduled within the range of dates. Second, more are requested to minimize the impact of expired observation windows, or failed observations. Windows of observation expire due to weather events or equipment failures at a particular site. At the end of the observation window, 50 observations were completed with only 9 failures.

\section{METHODS}

The photometric data produced by the Our Solar Siblings pipeline (M. Fitzgerald, 2018) was processed using Astrosource (M. T. Fitzgerald, Gomez, Salimpour,
Singleton, \& Wibowo, 2021). The first step in this process was to identify the exact galactic coordinates for the target star. This was accomplished by loading the .fits files for each respective filter into Aladin. Changing the frame to ICRSd for decimal coordinates gave us the required position to enter into Astrosource. This information must be entered with each filter to ensure the location of the star is correct for the rest of the program.

The images were then run through a filter to locate stars that could be seen in each picture with an acceptable brightness. The accepted range in counts by Astrosource was 2000-1000000. This was done to ensure there were brightness comparisons to use for the observed variable star (U Lep). The variability of the remaining stars was then measured. The goal was to identify the least variable of the remaining candidate stars to use as comparisons for U Lep. The location data for the least variable comparison stars was then used to identify known stars within the set. Depending on the bandpass, locations were cross-referenced with Skymapper (Wolf et al., 2018) or APASS (Henden et al., 2016) and the magnitude of the comparison stars was determined.

In order to obtain the light curve from the data set, the variability in magnitude was found. This was done by using the comparison stars to measure the difference in magnitude compared to the target star. The last part of the program calculates the period. This was done by testing several values for the period and looking for the most likely candidate. The data taken over the course of 12 days was tested for varying periods until a light curve could be found.

\section{RESULTS}

After taking data over the course of two weeks from October 02, 2019 to October 14, 2019, we confirmed the period of $U$ Lep to be 0.581 days with an error of 0.011 days. This value was calculated by taking the mean of our period and error measurements for each band. The resulting period was consistent with those reported in the literature, including Hipparcos 0.581474 (Agency, 1997), 0.581479 (Feast et al., 2008), and 0.5814762 days (Kazarovets, Durlevich, Kireeva, Pastukhova, et al., 2017). The final periods, as calculated for each bandpass, was noted in Table 2.

U Leporis completed 20.6 cycles of variation during the 12 day observation window. Astrosource generated four phased light curves from the data collected, 
Table 2. Period from each filter used.

\begin{tabular}{ccc}
\hline \hline Band & Period $(\mathrm{d})$ & Error $(\mathrm{d})$ \\
\hline B & 0.58276 & 0.01050 \\
V & 0.58006 & 0.01208 \\
i & 0.58106 & 0.01053 \\
z & 0.58101 & 0.01225 \\
\hline
\end{tabular}

as seen in Figure 2. The shape of each light curve (a sharp peak followed by a gradual decline) was consistent with those of other RRab-type RRL variables, which pulsate in the fundamental radial mode (Catelan \& Smith, 2015).

The resulting data lists several apparent magnitudes for each filter taken over the course of 12 days of observation. Table 3 shows the range and amplitude of apparent magnitudes for each band.

Table 3. Variation in apparent magnitude.

\begin{tabular}{ccccc}
\hline Band & Min & Mid & Max & Amp \\
\hline B & 11.53 & 10.75 & 9.96 & 1.57 \\
V & 11.08 & 10.55 & 10.01 & 1.06 \\
i & 10.82 & 10.30 & 9.77 & 1.04 \\
z & 10.76 & 10.35 & 9.93 & 0.83 \\
\hline
\end{tabular}

Table 3 and Figure 2 reveal two trends in the light curves related to bandpass. First, the minimum apparent magnitude brightens as the peak wavelength of the bandpass increases. This trend is consistent with the idea that galactic reddening and metallicity increase apparent magnitudes of visible bandpasses more than near infrared. Second, the amplitude of the variation decreases as the peak wavelength of the bandpass increases. Both trends are consistent with observations of other RRL (Catelan \& Smith, 2015).

Calibrations for V, i, and z (Catelan et al., 2004; Cáceres \& Catelan, 2008) and established metallicity $([\mathrm{Fe} / \mathrm{H}]=-1.93)$ (Dambis et al., 2013) were used to determine the distance to U Lep. Two methods were attempted and compared in order to account for reddening. The first of these, minimized variance, was based on the fact that the distance to U Lep should be the same regardless of filter. The value for color excess was adjusted until the standard deviation of
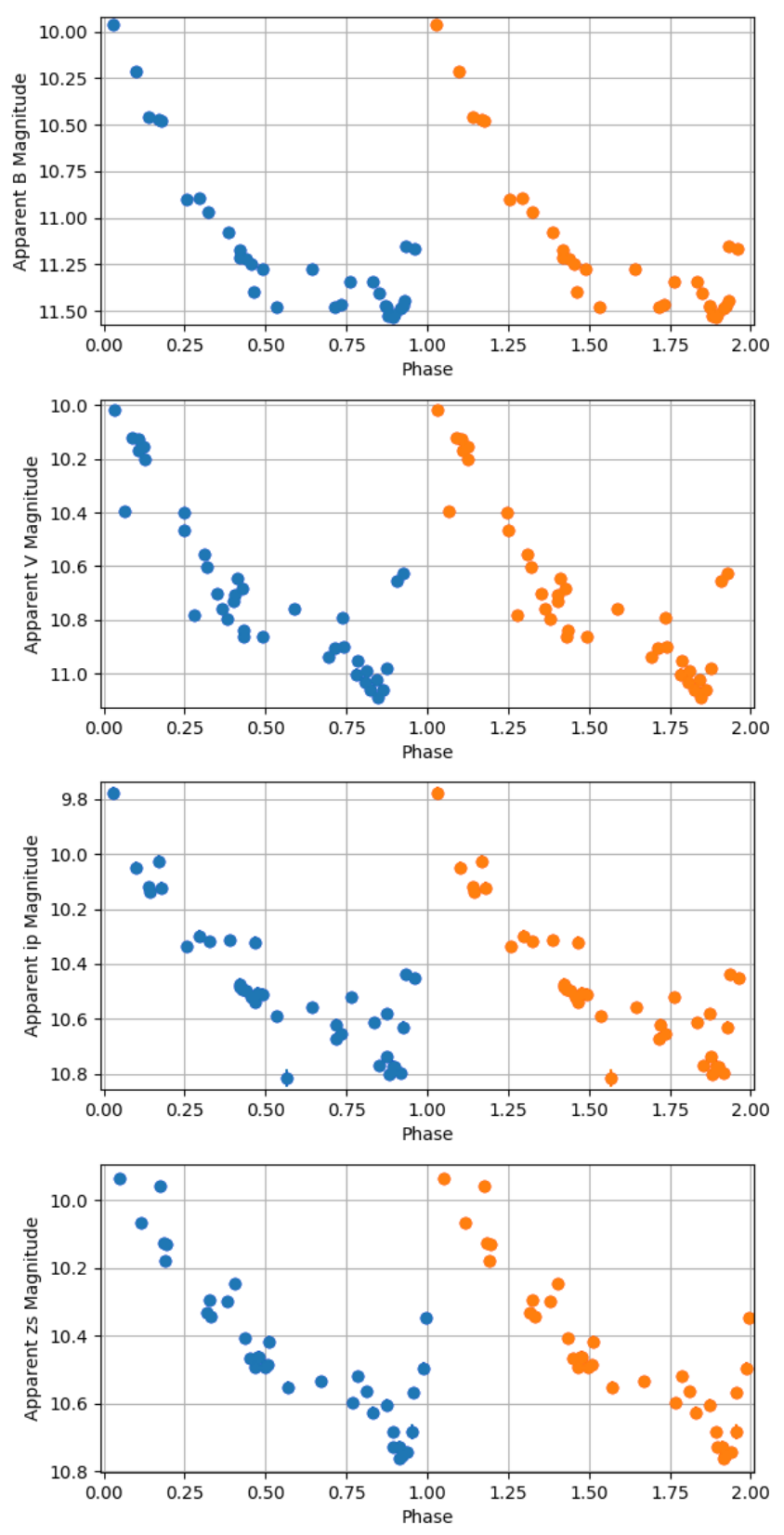

Fig. 2. Light curves taken from the four different bands that were observed. One 'phase' on the $\mathrm{x}$ axis is equivalent to the calculated period for each band respectively.

the distances predicted by calibrations of the V, i, z filters was minimized. Trial and error yielded a value of $\mathrm{E}(\mathrm{B}-\mathrm{V})=0.065$. The second method employed estimates of Galactic dust extinction of the Sloan Digital Sky Survey (Schlafly \& Finkbeiner, 2011). The mean color excess was determined to be $\mathrm{E}(\mathrm{B}-\mathrm{V})=0.0290$ $+/-0.0015$. This value was less than half the value suggested by the first method.

The distances determined for each bandpass and 
by each method are reported in Table 4 and shown visually in Figure 3. The average distances based on minimized variance and by mean galactic dust were determined to be $903 \pm 20$ pc and $938 \pm 21$ pc respectively.

Table 4. Distance to U Lep for each bandpass.

\begin{tabular}{ccccc}
\hline \hline & variance & \multicolumn{3}{c}{ SDSS } \\
\hline Band & Dist (pc) & Error (pc) & Dist (pc) & Error (pc) \\
\hline V & 926 & 37 & 968 & 38 \\
i & 904 & 33 & 932 & 33 \\
z & 930 & 37 & 951 & 38 \\
\hline AVG & 920 & 20 & 950 & 21 \\
\hline
\end{tabular}

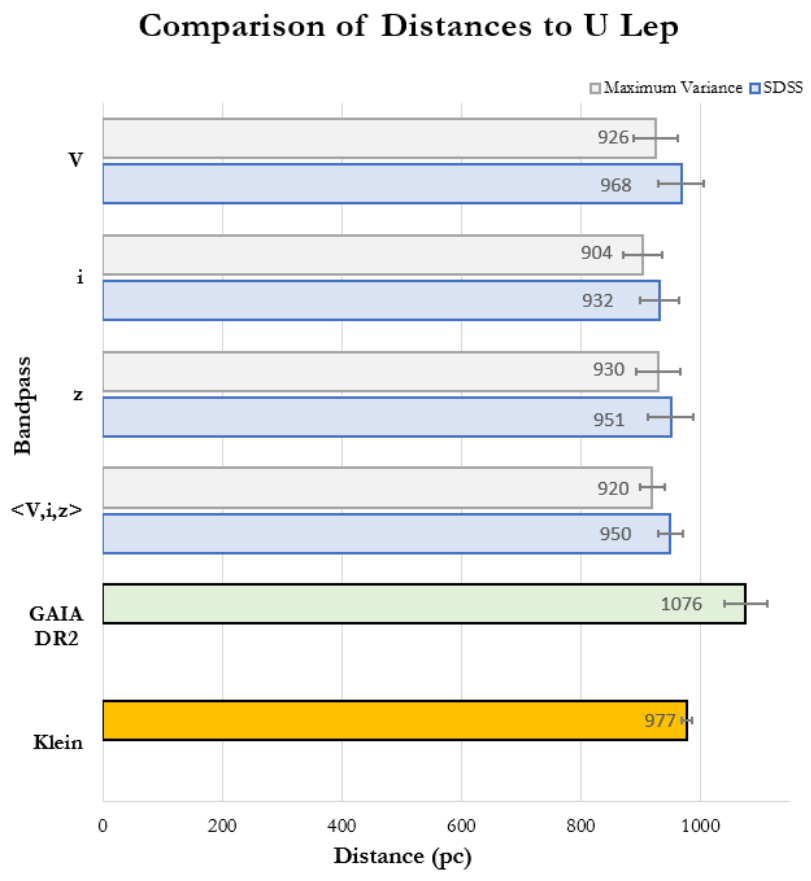

Fig. 3. Distances to U Lep for each bandpass using two different models for extinction.

\section{DISCUSSION}

The determined periods from light curves taken through four filters are consistent with one another and previously established values. The three calibration models for $\mathrm{V}, \mathrm{i}$, and $\mathrm{z}$ provided distances to $\mathrm{U}$ Lep that were inconsistent with one another and with Gaia and Klein.
Adjusting the color excess to minimize the variance reduced the inconsistencies within the filters but led to a reddening factor that exceeds estimates based on previous measurements of galactic dust by a factor of two. This method also widened the gap between the mean of the three distances and Gaia to 156 pc or 16 $\%$ closer to Earth.

Using the mean galactic reddening yielded an average distance only about $12 \%$ less than that determined by parallax using the Gaia DR2 data (1076 \pm $36 \mathrm{pc}$ ). The mean distance of $950 \pm 21 \mathrm{pc}$ was closer to the distance of $977 \pm 8.3$ pc determined by Klein (2011) using data from WISE. The distances for V and $\mathrm{z}$ are within one standard deviation of this mark.

The calibrations used in this study were based on the realization that the Horizontal Band is not horizontal toward the near-infrared i and $\mathrm{z}$ (Catelan et al., 2004; Catelan \& Smith, 2015) and thus a periodluminosity (PL) relationship could be determined:

$$
\begin{gathered}
M_{V}=2.288-0.882 \log Z+0.108(\log Z)^{2} \\
M_{i}=0.908-1.035 \log P+0.220 \log Z \\
M_{z}=0.839-1.295 \log P+0.211 \log Z
\end{gathered}
$$

where

$$
\log Z=[F e / H]+\log (0.638 f+0.362)
$$

and

$$
f=10^{3}
$$

The calibrations for $\mathrm{i}$ and $\mathrm{z}$ were determined through an extensive analysis of the Sloan Digital Sky Survey, with strong correlation coefficients for each $\left(r_{i}=0.95, r_{z}=0.97\right)$. For $U$ Lep the calculated period and calibrated magnitudes determined for $\mathrm{i}$ and $\mathrm{z}$ yielded distances well within the estimated error range, each of which are less than $4 \%$ of the determined distances.

One curiosity was that the observed apparent magnitudes did not follow the anticipated trend of increase as wavelength decreased. The middle magnitude of the $i$ bandpass (10.30) is slightly less than that measured for $\mathrm{z}$ (10.35). This quirk led the distance estimated based on the i bandpass (904 pc) to be the shortest of the three ( $926 \mathrm{pc}$ and $930 \mathrm{pc}$ for $\mathrm{V}$ and $\mathrm{z}$, respectively). The minimized variance method brought the $V$ and $z$ estimates to within $4 \mathrm{pc}$ of one another but failed to close the much larger gap between $\mathrm{i}$ and V $(22 \mathrm{pc})$. The trend in decreased amplitude of apparent magnitude with increasing wavelength was not disrupted, however the amplitude variations for $\mathrm{V}$ (1.06) and i (1.04) were almost identical. 


\section{CONCLUSION}

The four light curves generated for U Lep were consistent with those expected for class RRab stars and with previously reported observations of U Lep. The mean period of $0.581+/-0.011$ days was consistent with previously reported measurements. Distances to U Lep were calculated for each bandpass using calibrations for V (Catelan et al., 2004) and i and z (Cáceres \& Catelan, 2008). Magnitudes were adjusted for color excess using two methods: (1) minimizing variance and (2) mean galactic reddening based on the Sloan Digital Sky Survey. The first method yielded distances in parsecs for $\mathrm{V}, \mathrm{i}, \mathrm{z}$ of $926 \pm 37,904 \pm 33$, and $930 \pm 37$, for an average of $920 \pm 20$. The second method yielded distances in parsecs for V, i, z of $968 \pm 38,932 \pm 33$, and $951 \pm 38$, for an average of $950 \pm 21$. These distances to U Lep were closer to those determined using Hipparcos data adjusted for galactic reddening (Klein et al., 2011) than to the more precise parallax measures determined by Gaia (A. Brown et al., 2018).

The lack of agreement in the distances suggests errors either in the Gaia DD2 measures or in the empirically determined period-luminosity relationships for near infrared $\mathrm{i}$ and $\mathrm{z}$. The difference of $12 \%$ between the SDSS and Gaia DR2 measurements is greater than the error estimates would bridge but is not an obscene difference. Application of the two methods to a larger set of RRL variables is necessary to better understand the significance in the difference in estimated distances.

\section{ACKNOWLEDGMENTS}

This work makes use of observations from the LCOGT network. This research has made use of "Aladin sky atlas" (Bonnarel et al., 2000; Boch \& Fernique, 2014) and the SIMBAD database (Wenger et al., 2000) developed at CDS, Strasbourg Observatory, France. This research used Astrosource 1.1.1, developed by Michael Fitzgerald and Edward Gomez, to identify comparison stars in the images https://pypi.org/ project/astrosource.

\section{REFERENCES}

Agency, E. S. (1997). The hipparcos and tycho catalogues: astrometric and photometric star catalogues derived from the esa hipparcos space astronometry mission. 11. hipparcos variability an- nex: periodic and unsolved variables and spectral types. ESA Publ. Division.

Boch, T., \& Fernique, P. (2014). Aladin lite: Embed your sky in the browser. In Astronomical data analysis software and systems xxiii (Vol. 485, p. 277).

Bonnarel, F., Fernique, P., Bienaymé, O., Egret, D., Genova, F., Louys, M., ... Bartlett, J. G. (2000). The aladin interactive sky atlas-a reference tool for identification of astronomical sources. Astronomy and Astrophysics Supplement Series, 143(1), 33-40.

Brown, A., Vallenari, A., Prusti, T., De Bruijne, J., Babusiaux, C., Bailer-Jones, C., ... others (2018). Gaia data release 2-summary of the contents and survey properties. Astronomy \& astrophysics, 616, A1.

Brown, T., Baliber, N., Bianco, F., Bowman, B. B., M., Conway, P., Crellin, M., ... et al. (2013). Las cumbres observatory global telescope network. Publications of the Astronomical Society of the Pacific, 125, 1031-1055.

Catelan, M., Pritzl, B. J., \& Smith, H. A. (2004). The RR Lyrae period-luminosity relation. i. theoretical calibration..

Catelan, M., \& Smith, H. A. (2015). Pulsating stars. Wiley-VCH.

Clementini, G., Federici, L., Corsi, C., Cacciari, C., Bellazzini, M., \& Smith, H. A. (2001). Rr lyrae variables in the globular clusters of m31: a first detection of likely candidates. The Astrophysical Journal Letters, 559(2), L109.

Cáceres, C., \& Catelan, M. (2008, Nov). The periodluminosity relation of RR Lyrae stars in the SDSS photometric system. The Astrophysical Journal Supplement Series, 179(1), 242-248. Retrieved from http://dx.doi.org/10.1086/ 591231

Dambis, A. K., Berdnikov, L. N., Kniazev, A. Y., Kravtsov, V. V., Rastorguev, A. S., Sefako, R., \& Vozyakova, O. V. (2013, 09). RR Lyrae variables: visual and infrared luminosities, intrinsic colours and kinematics. Monthly Notices of the Royal Astronomical Society, 435(4), 3206-3220. Retrieved from https://doi.org/ $10.1093 / \mathrm{mnras} / \mathrm{stt} 1514$

Du, H., Mao, S., Athanassoula, E., Shen, J., \& Pietrukowicz, P. (2020). Kinematics of rr lyrae stars in the galactic bulge with ogle-iv and gaia dr2. Monthly Notices of the Royal Astronomical 
Society, 498(4), 5629-5642.

Feast, M. W., Laney, C. D., Kinman, T. D., Van Leeuwen, F., \& Whitelock, P. A. (2008). The luminosities and distance scales of type II Cepheid and RR Lyrae variables. Monthly Notices of the Royal Astronomical Society, 386(4), 2115-2134.

Fitzgerald, M. (2018). The our solar siblings pipeline: Tackling the data issues of the scaling problem for robotic telescope based astronomy education projects.

Fitzgerald, M. T., Gomez, E., Salimpour, S., Singleton, J., \& Wibowo, R. W. (2021). "astrosource": automating optical astronomy measurement, calibration and analysis for variable stellar sources from provided photometry. Journal of Open Source Software, 6(58), 2641.

Henden, A. A., Templeton, M., Terrell, D., Smith, T. C., Levine, S., \& Welch, D. (2016, January). VizieR Online Data Catalog: AAVSO Photometric All Sky Survey (APASS) DR9 (Henden+, 2016). VizieR Online Data Catalog, II/336.

Hernitschek, N., Cohen, J. G., Rix, H.-W., Sesar, B., Martin, N. F., Magnier, E., ... others (2018). The profile of the galactic halo from pan-starrs1 $3 \pi$ rr lyrae. The Astrophysical Journal, 859(1), 31.

Iorio, G., \& Belokurov, V. (2018, 10). The shape of the Galactic halo with Gaia DR2 RR Lyrae. Anatomy of an ancient major merger. Monthly Notices of the Royal Astronomical Society, 482(3), 3868-3879. Retrieved from https://doi.org/ 10.1093/mnras/sty2806 doi:

Kazarovets, E., Durlevich, O., Kireeva, N., Pastukhova, E., et al. (2017). General catalogue of variable stars: Version gcvs 5.1. Astronomy Reports, 61(1), 80-88.

Keller, S. C., Murphy, S., Prior, S., DaCosta, G., \& Schmidt, B. (2008). Revealing substructure in the galactic halo: the sekbo rr lyrae survey. The Astrophysical Journal, 678(2), 851.

Klein, C. R., Richards, J. W., Butler, N. R., \& Bloom, J. S. (2011). Mid-infrared period-luminosity relations of RR Lyrae stars derived from the WISE preliminary data release. The Astrophysical Journal, 738(2), 185.

Mateu, C., \& Vivas, A. K. (2018). The galactic thick disc density profile traced with rr lyrae stars. Monthly Notices of the Royal Astronomical Society, 479(1), 211-227.
Sarajedini, A., Barker, M., Geisler, D., Harding, P., \& Schommer, R. (2006). Rr lyrae variables in m33. i. evidence for a field halo population. The Astronomical Journal, 132(3), 1361.

Schlafly, E. F., \& Finkbeiner, D. P. (2011). Measuring reddening with sloan digital sky survey stellar spectra and recalibrating sfd. The Astrophysical Journal, 737(2), 103.

Soszyński, I., Udalski, A., Wrona, M., Szymański, M., Pietrukowicz, P., Skowron, J., . . others (2019). Over 78000 rr lyrae stars in the galactic bulge and disk from the ogle survey. arXiv preprint arXiv:2001.00025.

Wegg, C., Gerhard, O., \& Bieth, M. (2019, 02). The gravitational force field of the Galaxy measured from the kinematics of RR Lyrae in Gaia. Monthly Notices of the Royal Astronomical Society, 485(3), 32963316. Retrieved from https://doi.org/10 .1093/mnras/stz572 doi:

Wenger, M., Ochsenbein, F., Egret, D., Dubois, P., Bonnarel, F., Borde, S., ... Monier, R. (2000, Apr). The SIMBAD astronomical database. The CDS reference database for astronomical objects. aaps, 143, 9-22. doi:

Wolf, C., Onken, C. A., Luvaul, L. C., Schmidt, B. P., Bessell, M. S., Chang, S.-W., ... others (2018). Skymapper southern survey: first data release (dr1). Publications of the Astronomical Society of Australia, 35.

Wu, C., Qiu, Y., Deng, J., Hu, J., \& Zhao, Y. (2006). $[\mathrm{Fe} / \mathrm{H}]$ derived from the light curves of RR Lyrae stars in the Galactic halo. Astronomy \& Astrophysics, 453(3), 895-902. 